\title{
Sulfato de calcio: propiedades y aplicaciones clínicas
}

\author{
Calcium sulfate: properties and clinical applications
}

\author{
López J1 , Alarcón M²
}

\begin{abstract}
RESUMEN
Durante más de cien años el sulfato de calcio se ha distinguido de otros biomateriales, por ser uno de los materiales aloplásticos más simples y que presenta la más larga historia clínica como material sintético. Entre sus principales propiedades destacan que es de fácil obtención, capacidad de osteoconducción, es totalmente absorbible, no provoca reacciones de inflamación o antigénicas, fácil modelado y fuerza de compresión semejante al tejido óseo. Todas éstas características son necesarias para ser usado como sustituto óseo. Sin embargo, diferencias en la morfología y estructura de sus cristales, la porosidad, propiedades mecánicas y en la constitución química del sulfato de calcio puede alterar significativamente sus propiedades biológicas. El objetivo de la presente revisión es conocer las propiedades del sulfato de calcio y sus diferentes aplicaciones clínicas como sustituto óseo en procedimientos regenerativos.
\end{abstract}

Rev. Clin. Periodoncia Implantol. Rehabil. Oral Vol. 4(3); 138-143, 2011.

Palabras clave: Preservación del reborde, exodoncia, implantes dentales, injerto óseo, regeneración.

\section{ABSTRACT}

For over 100 years, the calcium sulfate has stood out from other biomaterials, for being one of the simplest alloplastic materials and having the longest history as synthetic material. It is readily available, has osteoconductive properties, is completely absorbed, does not cause inflammation or antigenic reactions, can be modeled to defect, has compressive strength similar to bone tissue and can be used as a vehicle for in situ release of chemotherapeutic agents. All these features are required to be used as a bone substitute. However, differences in morphology, crystal structure, porosity, mechanical properties and chemical constitution of calcium sulfate can significantly alter its biological properties. The aim of this study was to determine through a review of the literature, the properties of calcium sulfate and its various clinical applications as bone substitute in regenerative procedures.

Rev. Clin. Periodoncia Implantol. Rehabil. Oral Vol. 4(3); 138-143, 2011.

Key words: Alveolar ridge preservation, extraction, dental implants, bone grafting, regeneration.

\section{INTRODUCCIÓN}

El sulfato de calcio (SC) ocupa una posición única en el universo de materiales renovables. Tiene una historia más larga de uso clínico que la mayoría de los biomateriales disponibles en la actualidad y es ampliamente reconocido como un material bien tolerado, con aplicaciones en la regeneración ósea. Sufre casi reabsorción completa en vivo, sin provocar una significativa respuesta inflamatoria, una propiedad deseable compartida por unos pocos materiales sustitutos óseos(1).

La materia prima de la que se hace es relativamente barata y abundante, se puede combinar con otros materiales como autoinjertos, aloinjertos, fosfatos de calcio, y vidrios bioactivos, para aumentar sus propiedades de unión, el volumen y la eficacia de los injertos óseos. También ha servido como un material de vehículo excelente para los factores de crecimiento y múltiples drogas, a pesar de estas ventajas, el material no ha gozado de la popularidad de muchos otros materiales de regeneración, aunque recientemente ha recibido una atención renovada con propiedades hemostáticas, angiogénicas y de barrera o membrana para la preservación de rebordes alveolares postexodoncias $^{(1-8)}$.

\section{ANTECEDENTES}

Los primeros antecedentes históricos del uso del sulfato de calcio (SC) se remontan al periodo de los ancestrales egipcios, los cuales lo usaban para cubrir los vendajes que envolvían las momias. En el área médica, el sulfato de calcio ya era utilizado desde el siglo XII para contener las partes del cuerpo.

El primer reporte de su uso como material de injerto óseo fue por el médico alemán Friedrich Trendelenburg en Bonn. Luego Dreesman en 1892 en el tratamiento de ocho pacientes que presentaban defectos en los huesos largos del cuerpo, provocados por infección utilizando sulfato de calcio como sustituto óseo sintético y material de relleno de defectos óseos ${ }^{(9)}$. Posteriormente, Nielson en 1944 relató los resultados del tratamiento de 14 pacientes con defectos óseos no infectados y 16 pacientes con defectos óseos infectados; los resultados de la colocación de sulfato de calcio fueron satisfactorios en todos los $\operatorname{casos}^{(10)}$

Peltier y Lillo en 1955, fueron los primeros investigadores en estudiar los efectos del sulfato de calcio como material para implante en cavidades óseas. Los autores insertaron cilindros de sulfato de calcio, previamente fabricados en defectos quirúrgicamente elaborados

1. Diplomado en Periodoncia. Alumno del Programa de Especialización en Implantología Oral de la UPCH. Universidad Peruana Cayetano Heredia. Perú.

2. Especialista y Magíster en Periodoncia. Docente del Programa de Especialización en Implantología Oral de la UPCH. Universidad Peruana Cayetano Heredia. Perú.

Correspondencia autor: José Luis López Herrera. drluislopez40@hotmail.com. Residencial San Felipe. Magnolias Dpto. 603 Distrito de Jesús María. C.O.P. 6137. Lima, Perú. Trabajo recibido el 09/05/2011. Aprobado para su publicación el 10/08/2011. 
en 14 perros. Según los autores, los defectos preparados fueron de tamaño crítico, o sea, eran suficientemente grandes para impedir la reparación ósea espontánea. A los 45 y 72 días postoperatorios, fueron realizados los análisis radiográfico y microscópico, evidenciando una completa reabsorción del material. Los autores concluyeron que existió reparación ósea completa de los defectos que recibieron el sulfato de calcio a los tres meses postoperatorios ${ }^{(11)}$.

\section{PROPIEDADES DEL SULFATO DE CALCIO}

\section{Propiedades Físicas y Químicas del "Sulfato de Calcio o Yeso"}

Es un mineral compuesto de sulfato de calcio dihidratado $\left(\mathrm{CaSO}_{4} \cdot 2 \mathrm{H}_{2} \mathrm{O}\right)$. La materia prima se obtiene a través de diversos tipos de operaciones mineras. Antes de su uso para aplicaciones en medicina el sulfato de calcio debe ser examinado por sus impurezas, tales como: silicatos, estroncio, plomo y otros materiales de origen natural ${ }^{(14)}$.

Cuando el yeso se calienta a $110^{\circ} \mathrm{C}$, se pierde agua en un proceso conocido como la calcinación. El producto resultante es el sulfato de calcio hemihidratado, también conocido como yeso de Paris $^{(15)}$.

$$
\mathrm{CaSO}_{4} \cdot 2 \mathrm{H}_{2} \mathrm{O} \stackrel{\text { Calor }}{\longrightarrow} \mathrm{CaSO}_{4} \cdot 1 / 2 \mathrm{H}_{2} \mathrm{O}+1 \frac{1}{2} \mathrm{H}_{2} \mathrm{O}
$$

La forma del sulfato de calcio hemihidratado, existe en dos formas, una Alfa y una Beta, que difieren en tamaño de los cristales, superficie y red de imperfecciones. Aunque estos materiales son químicamente idénticos difieren considerablemente en sus propiedades físicas. La forma Alfa-hemihidratado es el yeso dental que se emplean en modelos de diagnóstico. Es muy duro y relativamente insolubles en comparación con el Betahemihidratado.

El Beta-hemihidratado se caracteriza por un conjunto de cristales irregulares con poros capilares intersticiales, mientras que el Alfa-hemihidrato contiene fragmentos de división y prisma de cristales en forma de barra(15). Cuando el hemihidratado se mezcla con agua, el dihidratado es formado en una leve reacción exotérmica.

$\mathrm{CaSO}_{4} \cdot 1 / 2 \mathrm{H}_{2} \mathrm{O}+1 \frac{1}{2} \mathrm{H}_{2} \mathrm{O} \longrightarrow \mathrm{CaSO}_{4} \cdot 2 \mathrm{H}_{2} \mathrm{O}+$ Calor

Biocompatibilidad y Propiedades Biológicas

La Biocompatibilidad es una condición sine qua non para materiales implantables y es el resultado de interacciones complejas en la interface huésped-material.

La falta de una respuesta del huésped posterior a la implantación es una característica importante de un material biocompatible. Muchos investigadores han observado una mínima respuesta inflamatoria posterior a la implantación de SC. Informes dentales y publicaciones ortopédicas indican que la reabsorción de SC es rápida y completa en comparación con otros materiales regenerativos implantables ${ }^{(8,11)}$

Las interacciones entre las células del huésped y un sustrato es otra forma de evaluar la biocompatibilidad. Payne y cols. evaluaron la capacidad en cultivos de fibroblastos gingivales humanos como migraron sobre diversos materiales de barrera en respuesta a un estímulo quimiotáctico ${ }^{(17)}$. Los materiales de barrera probados fueron de Politetrafluoretileno (ePTFE), membranas de ácido poliláctico (PLLA), y SC con poliestireno que sirvieron como control. Las células fueron capaces de migrar más en el sustrato de SC que en las otras barreras de regeneración tisular guiada a prueba. Examen microscópico electrónico de barrido indica que la morfología celular parecía normal en el substrato SC, mientras que las células en barreras de ácido poliláctico y ePTFE tenían una morfología anormal y no parecían estar migrando. Los autores sugieren que esta propiedad puede ser de especial importancia en sitios donde el cierre primario de la herida no puede ser alcanzado.

\section{Propiedades Regenerativas}

El sulfato de calcio usado como sustituto óseo es absorbido por disolución en ocho semanas, dependiendo del volumen y del sitio de implantación. A pesar de que no se han descrito en detalle los posibles mecanismos con los que el SC promueve la regeneración y reparación ósea existen explicaciones y observaciones plausibles de diferentes autores que consideradas en conjunto, pueden explicar su acción.

Strocchi y cols. crearon defectos óseos en la tibia de conejos, que fueron llenados con gránulos SC o hueso autógeno, la densidad microvascular se incrementó en los defectos tratados con SC, lo que sugiere un efecto positivo sobre la angiogénesis necesaria para promover la regeneración ósea ${ }^{(1)}$.

En el campo odontológico los investigadores Lebourg y Biou en 1961, implantaron sulfato de calcio en alvéolos después de la extracción de terceros molares inconclusos, así como en los otros defectos óseos en la mandíbula y maxilar. Después de tres a cuatro semanas se observó que la masa de sulfato de calcio se había reabsorbido completamente, y la reparación ósea había sido acelerada en las áreas tratadas en comparación con las áreas control. Los autores concluyeron que el sulfato de calcio constituía un material favorable para el tratamiento de defectos óseos y justificaron tal hecho por la habilidad del referido material en suplir iones inorgánicos fundamentales para el proceso de reparación ${ }^{(12)}$

Walsh y cols. utilizaron técnicas de inmunohistoquímica para identificar el crecimiento de varios factores de crecimiento en defectos femorales esponjosos llenos con bolitas de sulfato de calcio.

Se observó aumento de las concentraciones de las proteínas morfogenéticas óseas (BMP) -2, BMP-7, factor de crecimiento transformante-b (TGF-b), y el factor de crecimiento derivado de plaquetas (PDGF), todos los cuales desempeñan un papel en la regeneración del tejido conjuntivo. En conjunto, estos resultados sugieren que el SC no actúa simplemente como un relleno inerte, pero puede desempeñar un papel más activo en la osteogénesis ${ }^{(15)}$.

Otro evento importante del sulfato de calcio es que sufre una degradación en el defecto y una disminución local en el pH. Esta caída del $\mathrm{pH}$ produce una desmineralización de las paredes del defecto con liberación de factores de crecimiento óseo. Estudios recientes indican que hay una mayor expresión de proteína morfogenética ósea-2 (BMP-2), BMP-7, el TGF-beta y PDGF-BB en defectos óseos cuando sulfato de calcio se utiliza como un injerto de hueso. Todos estos factores de crecimiento estimulan la formación y el desarrollo de hueso nuevo. Originalmente el sulfato de calcio fue percibido sólo como relleno de defectos, pero tiene muchos estudios recientes demostrados que el sulfato de calcio es biocompatible, biodegradable, osteoconductivo, seguro y no es tóxico ${ }^{(8,13-16)}$.

Coetzee en 1980, afirmó que para cada molécula de sulfato de calcio absorbida, una molécula de hidroxiapatita es depositada en el área local. Entre tanto, la teoría más palpable para explicar la naturaleza osteoconductiva del sulfato de calcio es la habilidad de este material para promover una fuente de iones inorgánicos que estimulen el crecimiento óseo.

Aunque los iones inorgánicos suficientes para la reparación de los defectos puedan ser proporcionados por la reabsorción osteoclástica del hueso en los bordes de los defectos, esto puede no ocurrir cuando el defecto es grande y la pérdida de hueso es extensa. Consecuentemente el injerto de sulfato de calcio en defectos grandes puede acelerar la cicatrización permitiendo la presencia de osteoblastos funcionales y la producción suficiente de matriz orgánica. Una vez implantado el sulfato de calcio en el cuerpo, se disuelve en iones de calcio y sulfato. Los iones de calcio se combinan con los iones fosfato a partir de los fluidos del cuerpo para formar fosfato de calcio esto forma una red osteoconductiva de apatita biológica que estimula el crecimiento óseo en el defecto. Estudios de espectroscopia infrarroja mostró que el nuevo material depositado es principalmente hidroxiapatita carbonatada, que es similar a la apatita presente de forma natural en el hueso(26).

Los iones de calcio son liberados durante la disolución del SC. Aumentos locales en la concentración de iones de calcio puede afectar la génesis y función de los osteoblastos, y que puede actuar como un estímulo a la diferenciación de los osteoblastos. El metafosfato de Calcio se ha demostrado que estimula la diferenciación osteoblástica de las células del estroma de la médula ósea. 


\begin{tabular}{|l|c|c|c|c|c|}
\hline & AUTOINJERTO & ALOINJERTO & $\begin{array}{c}\text { SULFATO } \\
\text { DE CALCIO }\end{array}$ & $\begin{array}{c}\text { FOSFATO } \\
\text { DE CALCIO }\end{array}$ & $\begin{array}{c}\text { GLASS } \\
\text { BIOACTIVO }\end{array}$ \\
\hline OSTEOCONDUCTIVO & SÍ & SÍ & SÍ & SÍ & SÍ \\
\hline BIOCOMPATIBLE & SÍ & SÍ & SÍ & SÍ & SÍ \\
\hline DEGRADACIÓN & LENTA & LENTA & COMPLETO & LENTA & LENTA \\
\hline HEMOSTÁTICO & NO & NO & SI & NO & NO \\
\hline ANGIOGÉNICO & DÉBIL & NO & FUERTE & NO & NO \\
\hline BARRERA-MEMBRANA & NO & NO & SÍ & NO & NO \\
\hline $\begin{array}{l}\text { LIBERACIÓN } \\
\text { FACTOR CRECIMIENTO }\end{array}$ & NO & NO & SÍ & NO & NO \\
\hline DISPONIBILIDAD & LIMITADA & ILIMITADA & ILIMITADA & ILIMITADA & ILIMITADA \\
\hline $\begin{array}{l}\text { TRANSMISIÓN } \\
\text { ENFERMEDAD }\end{array}$ & NO & POSIBLE & NO & NO & NO \\
\hline
\end{tabular}

Publicado por Osseonews. Dental Implant Information and Discussion. 1 de abril del 2008.

Ref: www.osseonews.com/dentogen-a-new-approach-to-bone-graftin.

El potencial de la membrana de sulfato de calcio para promover la regeneración ósea guiada fue evaluado por Pecora y cols. ${ }^{(22)}$ Defectos óseos quirúrgicos bicorticales fueron empleados en el ángulo de la mandíbula de ratas. En las cavidades de prueba se colocaron discos de sulfato de calcio pre endurecidos como barreras para cubrir los defectos. Las cavidades control permanecieron descubiertas.

Los periodos de análisis de los especímenes fueron de tres, nueve, 18 y 22 semanas postoperatorias. La evaluación microscópica demostró que en tres semanas postoperatorias, todas las cavidades cubiertas con la barrera de sulfato de calcio se mostraron total o parcialmente reparadas por tejido óseo. En los periodos tardíos, la mayor parte de las cavidades experimentales se encontraba rellena de hueso. El grupo control, sin embargo, mostró ausencia de formación ósea en tres y nueve semanas postoperatorias y reparación ósea parcial en 18 y 22 semanas postoperatorias. Los autores concluyeron que el sulfato de calcio fue efectivo como barrera, en función que impidió el crecimiento de tejido conjuntivo para el interior de las cavidades y posibilitó la neoformación ósea.

\section{APLICACIONES CLÍNICAS}

\section{Sulfato de Calcio en Defectos Óseos}

Dreesman fue el primero en reportar el uso de SC como material regenerativo en el siglo $\mathrm{XIX}^{(9)}$. Más de 60 años después, Lillo y Peltier ${ }^{(11)}$ utiliza barras de SC para reparar defectos óseos en un modelo canino e informó que el SC no promovió crecimiento de los huesos que estén cubiertos por periostio.

Sin embargo, cuando el periostio estuvo presente, hubo desaparición completa de SC de 45 a 72 días y la regeneración completa de los defectos en aproximadamente 3 meses. También tomó nota de la ausencia de inflamación, presencia de normalidad, y la morfología del hueso relacionada con células.

La ausencia de la inflamación y la reabsorción completa son recurrentes observaciones en la literatura. Radentz y Collings ${ }^{(18)}$ evaluando injertos de SC en defectos intraóseos en un modelo canino, en comparación con los controles no injertados.

Prueba y control de defectos curaron sin problemas, pero SC parecía el "sello" en el sitio, que protegía el sitio y evita hacia abajo el crecimiento del epitelio en la herida. Examen histológico reveló que el hueso en los defectos con SC, había un patrón trabecular más denso a las 12 semanas. Estos resultados en paralelo reportados por Bahn, quien sugirió SC fue "beneficioso para la regeneración ósea".

Nick M. Tovar y cols., colocaron sulfato de calcio de grado médico hemihidratado con hueso liofilizado en un canino con defecto periodontal profundo de un paciente con enfermedad periodontal avanzada para reparar y regenerar el defecto. El análisis de las radiografías a los 5 meses mostró que el hueso se había regenerado completamente(13).

\section{Como Injerto y Membrana o Barrera}

El uso del sulfato de calcio como barrera fue propuesto inicialmente por Sottosanti en 1992. Este autor propuso un protocolo de tratamiento combinando el sulfato de calcio con aloinjerto óseo desmineralizado seco y congelado (AODSC) como material para relleno de defectos óseos, asociados con una barrera de sulfato de calcio. En este estudio, el autor relató los resultados de cuatro casos clínicos que fueron tratados con el injerto de sulfato de calcio con el AODSC asociados a la barrera de sulfato de calcio. Estos materiales fueron usados en lesiones infraóseas periodontales en el relleno del alvéolo inmediatamente después de la extracción dental, y en la inmovilización inicial de un implante colocado en un alvéolo inmediatamente después a la extracción dental. El autor afirmó que el sulfato de calcio usado como barrera retardó la migración de los tejidos epitelial y conjuntivo para el interior de las lesiones, enfatizó la necesidad de estudios clínicos controlados para analizar detalladamente y comparar esta terapia con otras $^{(19)}$.

Sulfato de Calcio Asociado a Otros Biomateriales

Andreana y cols. en el 2004, compararon clínica y biológicamente el uso del sulfato de calcio asociado o no al aloinjerto óseo desmineralizado seco y congelado (AODSC) en procedimientos de levantamiento del seno maxilar, en seis pacientes para posteriormente rehabilitarse con implantes. Se realizaron biopsias óseas a los dos, seis y 24 meses postoperatorios. Los resultados microscópicos demostraron crecimiento óseo con pocos remanentes de AODSC. Los implantes fueron instalados en el momento del levantamiento del seno maxilar o después de seis meses postoperatorios. Los casos relatados indicaron que el sulfato de calcio puede ser usado puro o asociado al AODSC con éxito en procedimientos de levantamiento del seno maxilar ${ }^{(20)}$

Anson $^{(21)}$, en 1996, relató la experiencia clínica con cuatro casos de pacientes tratados con la técnica propuesta por Sottosanti. El autor implantó el complejo de sulfato de calcio y AODSC asociados a la barrera de sulfato de calcio en defectos óseos periodontales. Los casos fueron acompañados con exámenes radiográficos que revelaron aumento de radiopacidad de todas las regiones tratadas.

El autor realizó una serie de consideraciones enalteciendo las cualidades del sulfato de calcio empleado como barrera(21). Estos resultados fueron corroborados por Conner en 1996, en otro estudio clínico.

Kim y cols., resolvieron evaluar clínicamente el uso de la asociación del compuesto de sulfato y AODSC con la barrera de sulfato de calcio en defectos periodontales. Los pacientes se dividieron en dos grupos. Un grupo (experimental) fue tratado con la implantación de compuesto de sulfato de calcio y AODSC con barrera de sulfato de calcio.

En el grupo control, los defectos óseos periodontales fueron tratados con desbridamiento a campo abierto. Las medidas de la profundidad del sondeo, nivel de inserción clínica y profundidad de 
sondaje ósea fueron evaluadas en el preoperatorio y a los seis y 12 meses postoperatorios. Los autores concluyeron que la implantación de sulfato de calcio con AODSC asociados a una membrana de sulfato de calcio resultó en una significante mejoría en los parámetros clínicos de defectos óseos periodontales comparada al desbridamiento a campo abierto ${ }^{(23)}$

Couri y cols. en el 2002, compararon clínicamente la efectividad entre dos terapias regenerativas tisulares en defectos de furca clase II, en humanos. Treinta pares de defectos de furca clase II de molares inferiores de 13 pacientes fueron tratados aleatoriamente, con injertos de AODSC asociado a la membrana de politetrafluoretileno expandido (ePTFE) o con injerto de AODSC asociado a la barrera de sulfato de calcio. Los parámetros clínicos evaluados comprendieron profundidad del sondeo, cantidad de tejido queratinizado, dimensión de las recesiones y dimensiones de los defectos infraóseos en el transoperatorio. Los datos clínicos fueron evaluados antes, durante el tratamiento y después a los seis y 12 meses postoperatorios. Los autores concluyeron que ambos tratamientos proporcionaron un relleno significante del defecto a los seis meses postoperatorios. Entre tanto, enfatizaron que los defectos tratados con injerto de AODSC asociado a la membrana de ePTFE, presentaron un mayor relleno de hueso horizontal cuando se compararon con los que recibieron injerto de AODSC asociado a la barrera de sulfato de calcio. Los resultados contradictorios observados en los estudios clínicos y biológicos con la utilización del sulfato de calcio, son constantes y han sido atribuidos, particularmente, a la falta de criterios en la selección de la composición del material utilizado(24)

Frame $^{(25)}$ afirmó que los trabajos que habían sido realizados con el sulfato de calcio hasta 1975, evaluaron cuerpos sólidos del sulfato de calcio dihidratado con pocas informaciones sobre su pureza, preparación y esterilización. Hizo críticas también al método de esterilización de sulfato de calcio utilizado en las investigaciones hasta entonces que convertía la forma dihidratada en hemihidratada. El autor indicó cuales deberían ser las características ideales del sulfato de calcio, este debería ser preparado en la forma de partículas porosas, dihidratado, con alto grado de pureza. Así, el autor implantó esta forma de sulfato de calcio en subcutáneo de ratas. Observó que el injerto se reabsorbió más rápido y pudiera tener algún efecto conductor en la reparación ósea. El término sulfato de calcio se refiere a la sal cristalina que puede presentarse en varios grados de hidratación, o sea, el grado en que el agua está asociada a la estructura cristalina. El término yeso se refiere a un mineral natural común que se presenta en la forma dihidratada, o sea, $\mathrm{CaSO}_{4}$ $2 \mathrm{H}_{2} \mathrm{O}$.

La forma menos hidratada del sulfato de calcio, conocida como hemihidratada, posee la fórmula CaSO $1 / 2 \mathrm{H}_{2} \mathrm{O}$. Esta forma es obtenida sometiendo la forma dihidratada a un proceso de calentamiento conocido como calcinación, el proceso de calcinación realizado en autoclave a vapor de presión produce la forma $\alpha$-hemihidratada, caracterizada por estar formada por cristales de forma prismática y es la principal constituyente del yeso usado para la confección de modelos dentales en prótesis. El proceso de calcinación realizado al aire libre, a temperatura de 110 a $120^{\circ} \mathrm{C}$, produce la forma $\beta$-hemihidratada que contiene cristales de forma irregular conocido como yeso Paris usado como injerto.

\section{Sulfato de Calcio en Aumento de Seno Maxilar}

Dario De Leonardis y Gabriele E. Pecora en el año 2000, en un estudio prospectivo sobre el aumento del seno maxilar utilizando sulfato de calcio hemihidratado como material de injerto óseo en procedimientos de elevación de seno maxilar de 57 pacientes, divididos en 2 grupos, primer grupo control de 15 senos se coloco sulfato de calcio de forma no estratificada, y el segundo grupo test de 50 senos, grupo que se colocó el sulfato de calcio en forma de masilla por capas endurecidas con la finalidad de lograr formación de hueso vital para permitir la integración de los tejidos con los implantes endo-óseos y apoyar las restauraciones de prótesis en desdentados del maxilar posterior. Estos resultados y otros revelan buena formación de tejido clínica y radiográficamente ${ }^{(27)}$.

La técnica utilizada en el grupo de prueba produjo una reducción en la contracción del injerto durante la cicatrización, disminuyendo la tasa de reabsorción del material. Histológicamente, formación de nuevo hueso laminar con la maduración progresiva se encontró en ambos grupos.
Las muestras del grupo control mostraron histomorfométricamente un promedio de densidad ósea de $34.25 \pm 10.02 \%$, mientras que las muestras del grupo de prueba mostraron un valor promedio de $55.54 \pm 19.82 \%$, las conclusiones indicaron que el sulfato de calcio parece ser un materia efectivo para aumento de seno maxilar y la formación de hueso vital es propicio para la integración del implante. Además, la técnica utilizada en el grupo de prueba parecía mejorar la calidad y cantidad de la formación ósea.

\section{Preservación del Reborde Alveolar Post-Extracción Dental}

Wright HB, en el 2004, examinó la eficacia de Injertos de SC en forma de la raíz para preservación de la cresta del diente seguido de la extracción dental, un total de 44 sitios de extracción fueron estudiados, 22 recibieron el injerto y 22 sirvieron como control. Las inserciones fueron preformadas de forma estéril, de sulfato de calcio de grado médico y con una configuración parecida a la morfología de los dientes. Las inserciones se colocaron en los sitios de extracción después de la extirpación de dientes. Los sitios que tenían sulfato de calcio la preservación fue significativamente mayor en altura y anchura ósea que los controles no injertados. Las diferencias promedio de ambas dimensiones fueron de aproximadamente $1 \mathrm{~mm}^{(28)}$.

Shi y cols., en el 2007, utilizaron sulfato de calcio con y sin Plasma rico en plaquetas (PRP) como un relleno en sitios de extracción en un modelo canino. La adición de PRP mejora la cicatrización temprana de las heridas, pero no fue significativamente mejor que el SC solo. Ambos grupos con $\mathrm{SC}$ fueron superiores a la extracción que el grupo sin relleno(29).

Rolph CW, en el 2000, utiliza una técnica con un injerto de composite de Glass bioactivo y sulfato de calcio BG/SC, que fue colocado en alvéolos de extracción y posteriormente, cubierto con una capa de SC. Informó resultados positivos con esta técnica en cuanto a la conservación del ancho del reborde alveolar y una menor extensión en altura(30).

Vance GS y cols., en el 2004, utilizaron un injerto putty, masilla combinada con sulfato de calcio (un hemihidrato) y carboximetilcelulosa de sodio (CMC) para preservar las dimensiones del reborde alveolar en dientes extraídos.

Los sitios fueron cubiertos con una barrera de SC después de colocada la masilla experimental en los alvéolos. La masilla se comparó con un xenoinjerto de origen bovino cubiertos con una membrana de colágeno. Cambios dimensionales Post-extracción fueron similares en ambos grupos, pero fue significativamente hueso más vitales en el grupo putty de masilla experimental $(61 \%)$ frente al grupo xenoinjerto $(26 \%)^{(31)}$.

Otros clínicos ${ }^{(32,33)}$, han informado de resultados positivos cuando han utilizado sulfato de calcio como un material para la preservación de reborde en forma de relleno y de barrera en procedimientos de postextracción, proporciona una barrera que estabiliza el coágulo y excluye a los tejidos no deseados (por ejemplo, tejido epitelial, gingival y conectivo). Ayudando a cicatrizar y regenerar la zona para la colocación del implante adecuado, eliminando cualquier necesidad de posteriores injertos en bloque, o alguna otra forma de aumento del reborde, proporcionando un ahorro tanto para el paciente en tiempo y dinero. John Sottosanti(19), MD, y David Anson ${ }^{(21)}$, DDS, sostienen que una combinación de un injerto de hueso que contiene hueso liofilizado desmineralizado aloinjerto (DFDBA) y sulfato de calcio de grado médico hemihidratado aumenta el grado de calidad y cantidad de formación de hueso nuevo, tanto en alvéolos de extracción y defectos periodontales.

\section{CONCLUSIONES}

- El sulfato de calcio es biocompatible, biodegradable, osteoconductivo y seguro.

No toxico, angiogénico, hemostático y tiene propiedades de barrera o membrana.

Se puede combinar con autoinjertos, aloinjertos, fosfatos de calcio, y vidrios bioactivos. 
Sirve como un vehículo, excelente para la entrega de los factores de crecimiento, moléculas pequeñas, medicamentos y antibióticos.

- $\quad$ Se reabsorbe completamente en un período de tiempo relativamente corto, proporcionando un andamiaje que favorece la formación y crecimiento óseo. Proporciona una fuente abundante de iones de calcio que puede estimular la actividad osteoblástica.

- $\quad$ Actualmente usado como material de relleno y de barrera para la preservación de reborde alveolar para la colocación de implantes y en defectos óseos.

- $\quad$ Es relativamente barato.

- $\quad$ Cuando no se emplean las proporciones adecuadas durante la preparación y aplicación clínica de la pasta una rápida reabsorción puede en algunos casos ser perjudicial, la sangre puede interferir con la reacción de fraguado y tener un efecto adverso sobre las propiedades físicas del material.

- $\quad$ El material no es particularmente fuerte, incluso cuando las formas pre-establecidas se utilizan, esto hace que sea difícil cumplir con un relleno óseo total en las aplicaciones regenerativas.

\section{ESTUDIOS FUTUROS}

La investigación futura debe mejorar las características de manejo y las propiedades físicas del material, mientras se preserva su biocompatibilidad. Un Aumento en el costo beneficio de la bioactividad del sulfato de calcio puede ofrecer beneficios adicionales para su utilización.

Sin embargo, torna imperativo que las investigaciones caractericen y documenten adecuadamente la naturaleza del sulfato de calcio estudiado, de forma que los estudios puedan ser comparados sistemáticamente.

\section{CONFLICTOS DE INTERÉS} ningún tipo.

Los autores declaran no presentar conflictos de interés de

\section{REFERENCIAS BIBLIOGRÁFICAS}

1. Strocchi R, Orsini G, lezzi G, Scarano A, Rubini C, Pecora G, Piattelli A. Bone regeneration with calcium sulfate: Evidence for increased angiogenesis in rabbits. J Oral Implantol, 2002; 28: 273278.

2. Rosenblum SF, Frenkel S, Ricci JR, Alexander H. Diffusion of fibroblast growth factor from a plaster of Paris carrier. J Appl Biomater, 1993; 4: 67-72.

3. Nyan M, Sato D, Oda M, Machida T, Kobayashi H, Nakamura T, Kasugai $S$. Bone formation with the combination of simvastatin and calcium sulfate in critical-sized rat calvarial defect. J Pharmacol Sci, 2007; 104: 384-386.

4. Intini GE III. Engineering a bioactive matrix by modifications of calcium sulfate. Tissue Eng, 2002; 8: 997-1008.

5. Intini G, Andreana S, Intini FE, Buhite RJ, Bobek LA. Calcium sulfate and platelet-rich plasma make a novel osteoinductive biomaterial for bone regeneration. J Transl Med, 2007; 5: 13.

6. Bateman J, Intini G, Margarone J, Goodloe S, Bush P, Lynch SE, Dziak R. Platelet-derived growth factor enhancement of two alloplastic bone matrices. J Periodontol, 2005; 76: 1833-1841.

7. Kim SG, Chung CH, Kim YK, Park JC, Lim SC. Use of particulate dentin-plaster of Paris combination with/without platelet-rich plasma in the treatment of bone defects around implants. Int J Oral Maxillofac Implants, 2002; 17: 86-94.

8. Podaropoulos L, Veis AA, Papadimitriou S, Alexandridis C, Kalyvas. Bone regeneration using b-tricalcium in a calcium sulfate matrix. Journal of Oral Implantology, 2009; 35(1): 28-36.

9. Dreesman H. Uber Knochenplombierung. Beitr Klin Chir, 1892; 9: 804-810. 10. Nielson $A$. The filling of infected and sterile bone cavities by means of plaster of Paris. Act Chir Scand, 1944; 91: 17-27.

11. Peltier LF, Lillo R. The substitution of plaster of Paris rods for portions of the diaphysis of the radius in dogs. Surg Forum, 1955; 6: 556-558.

12. Lebourg L, Biou $C$. The imbedding of plaster of Paris in surgical cavities of the jaws. Sem Hop, 1961; 37: 1195-1197.

13. Nick M. Tovar, Ziv Mazor, Sachin Mamidwar, John L. Ricci. Reparación ósea en defectos periodontales el uso de un compuesto de aloinjerto y sulfato de calcio (DentoGen) como barrera. Journal of Oral Implantology Orim, 37-02-01.3d 2011.

14. Ricci JL, Alexander $H$, Nadkarni $P$, Hawkins $M$, Turner J, Rosenblum SF, Brezenoff L, De Leonardis D, Pecora G. Biological mechanisms of calcium-sulfate eplacement by bone. In: Davies JE, editor. Bone Engineering. Toronto: Em Squared Inc.; 2000. pp 332344.

15. Walsh WR, Morberg P, Yu Y, Yang JL, Haggard W, Sheath PC, Svehla $\mathrm{M}$, Bruce WJ. Response of a calcium sulfate bone graft substitute in a confined cancellous defect. Clin Orthop, 2003: 228-236.
16. Lazary A, Balla B, Kosa JP, Bacsi K, Nagy Z, Takacs I,Varga PP, Speer $G$, Lakatos $P$. Effect of gypsum on proliferation and differentiation of MC3T3-E1 mouse osteoblastic cells. Biomaterials, 2007; 28: 393399.

17. Payne JM, Cobb CM, Rapley JW et al. Migration of human gingival fibroblasts over guided tissue regeneration barrier materials. J Periodontol, 1996; 67: 236-244.

18. Radentz WH, Collings $\mathrm{CK}$. The implantation of plaster of paris in the alveolar process of the dog. J Periodontol, 1965; 36: 357-364.

19. Sottosanti JS. Aesthetic extractions with calcium sulfate and the principles of guided tissue regeneration. Pract Periodont Aesthet Dent, 1993; 5: 61-69.

20. Andreana S, Cornelini R, Edsberg LE, Natiella JR. Maxillary sinus elevation for implant placement using calcium sulfate with and without DFDBA: Six cases. Implant Dent, 2004; 13(3): 270-277.

21. Anson D. Calcium sulfate: A four-year observation of its use as a resorbable barrier in guided tissue regeneration of periodontal defects. Compend Contin Educ Dent, 1996; 17: 895-899.

22. Pecora G, Andreana S, Margarone III JE, Covani U, Sottosanti JS. Bone regeneration with a calcium sulfate barrier. Oral Surg Oral Med Oral Pathol Oral Radiol Endod, 1997; 84(4): 424-429.

23. Kim CK, Chai JK, Cho KS, et al.Periodontal repair in intrabony defects treated with a calcium sulfate implant and calcium sulfate barrier. J Periodontol, 1998; 69: 1317-1324.

24. Couri CJ, Maze GI, Hinkson DW, Collins BH 3rd, Dawson DV. Medical grade calcium sulfate hemihydrate versus expanded polytetrafluoroethylene in the treatment of mandibular class II furcations. $J$ Periodontol, 2002; 73(11): 352-359.

25. Frame JW. A composite of porous calcium sulphate dehydrate and cyanoacrylate as a substitute for autogenous bone. J Oral Surg, 1980; 38: 251-256.

26. Coetzee AS. Regeneration of bone in the presence of calcium sulfate. Arch Otolaryngol, 1980; 106: 405-409.

27. De Leonardis D, Pecora GE. Prospective study on the augmentation of the maxillary sinus with calcium sulfate: histological results. J Periodontol, 2000; 71: 940-947.

28. Wright HB. Ridge Preservation using pre-formed, root-shaped calcium sulfate inserts. Lexington, KY: University of Kentucky College of Dentistry; 2004: 62.

29. Shi B, Zhou Y, Wang YN, Cheng XR. Alveolar ridge preservation prior to implant placement with surgical-grade calcium sulfate and platelet-rich plasma: A pilot study in a canine model. Int $\mathrm{J}$ Oral Maxillofac Implants, 2007; 22: 656-665.

30. Rolph CW. Ridge preservation using bioactive glass and a calcium sulfate barrier. Lexington, KY: University of Kentucky College of Dentistry; 2000. 62p. 
31. Vance GS, Greenwell H, Miller RL, Hill M, Johnston H, Scheetz JP. Comparison of an allograft in an experimental putty carrier and a bovine-derived xenograft used in ridge preservation: A clinical and histologic study in humans. Int J Oral Maxillofac Implants, 2004; 19: 491-497.

32. Guarnieri R, Pecora G y cols. Medical grade calcium sulfate hemihydrate in healing of humam extraction sockets: Clinical and histological observations at 3 months. J Periodontal, 2004; 75: 902908.
33. Aimetti M, Romano F, Baima Griga F, Godio L. Clinical and histologic healing of human extraction sockets filled with calcium sulfate. Int J Oral Maxillofac Implants, 2009; 24: 901-909. 